11 Holtz TH, Sternberg M, Kammerer S, et al. Time to sputum culture conversion in multidrug-resistant tuberculosis: predictors and relationship to treatment outcome. Ann Intern Med 2006; 144: 650-659.

12 Leimane V, Dravniece G, Riekstina V, et al. Treatment outcome of multidrug/extensively drug-resistant tuberculosis in Latvia, 2000-2004. Eur Respir J 2010; 36: 584-593.

13 Kurbatova EV, Taylor A, Gammino VM, et al. Predictors of poor outcomes among patients treated for multidrug-resistant tuberculosis at DOTS-plus projects. Tuberculosis (Edinb) 2012; 92: 397-403.

14 Diacon AH, von Groote-Bidlingmaier F, Donald PR. Delamanid, a new 6-nitro-2,3-dihydroimidazo[2,1-b] oxazole for the management of tuberculosis resistant to at least isoniazid and rifampicin. Expert Opin Orphan Drugs 2014; 2: 87-94.

15 Falzon D, Jaramillo E, Schünemann HJ, et al. WHO guidelines for the programmatic management of drug-resistant tuberculosis: 2011 update. Eur Respir J 2011; 38: 516-528.

\title{
Is 1-year follow-up adequate for adult tuberculosis contacts?
}

\author{
To the Editor:
}

To eliminate tuberculosis (TB) on a global scale, the identification and neutralisation of latently infected high-risk individuals is of paramount importance [1]. Active TB contacts are well documented with an increased risk for both latent TB infection (LTBI) and the development of active TB disease [2]. Contact investigation is an important and effective active case-finding strategy, but also requires abundant public health resources [3].

The current World Health Organization (WHO) recommendation for contact investigation suggests that follow-up screening should be considered particularly in the first year after exposure, such as after 6 or 12 months [4]. However, most of TB contacts studies have included both adults and children, or enrolled children only. It remains uncertain if the 1-year follow-up period is adequate for all TB contacts [5]. To optimise the contact investigation strategy for adult TB contacts, understanding the dynamic pattern of active TB development is important. Characterising the clinical features of adult TB contacts with a higher risk for active TB progression is helpful to prioritise medical resources more efficiently. It also enables researchers to select individuals who will benefit the most from LTBI treatment in resource-limited areas.

To elucidate the issue, we conducted a nationwide population-based cohort study using data retrieved from the National Health Insurance Research Database (NHIRD) in Taiwan between January 2000 and December 2011. TB contacts were identified by diagnostic codes (V01.1 in the International Classification of Diseases (ICD), 9th revision, and the clinical modification ICD-9-CM) in conjunction with chest plain film radiography and/or sputum acid-fast smear examination and/or tuberculin skin test (TST). We also matched each TB contact with four nonexposed subjects by age and sex, and presence of comorbidities on the same index date of diagnosis. The TB contacts cohort and the matched cohort were followed until the development of active TB disease, which was defined by compatible ICD-9-CM codes (010-018 in ICD-9-CM) [6, 7]. The diagnosis of TB was validated by the prescription of at least two anti-TB medications for more than 28 days. If no TB occurred, subjects were followed until death, or the end of the study period (December 2011). Incidence rates (per 100000 person-years) of active TB were analysed, and hazard ratios were calculated as compared with matched controls. For multivariate adjustment, the Cox proportional hazards model was used to compute hazard ratios (HRs) and 95\% confidence intervals. All statistical analyses were performed using SAS 9.2 software (SAS Institute Inc., Cary, NC, USA).

A total of 8659 TB contacts were identified during the study period. Their median age was 44 years old, $13.8 \%$ were $\geqslant 65$ years of age, and $44.2 \%$ of them were male. This cohort was matched with 34636 individuals without TB contact history in terms of age, sex and major comorbidities. The median (interquartile range (IQR)) follow-up times for TB contacts and their matched controls were 2.25 (1.06-3.69) years and 2.27 (1.07-3.70) years, respectively. Overall, $72 \mathrm{~TB}$ contacts and 67 individuals from the matched cohort were documented with active TB during the follow-up period, and the mean annual incidences of TB were 312.8 and 72.2 cases per 100000 person-years, respectively (adjusted HR 4.39, 95\% CI 3.15-6.12; p<0.001). The Kaplan-Meier analysis and annual incidences of active TB in TB contacts and control participants are shown 


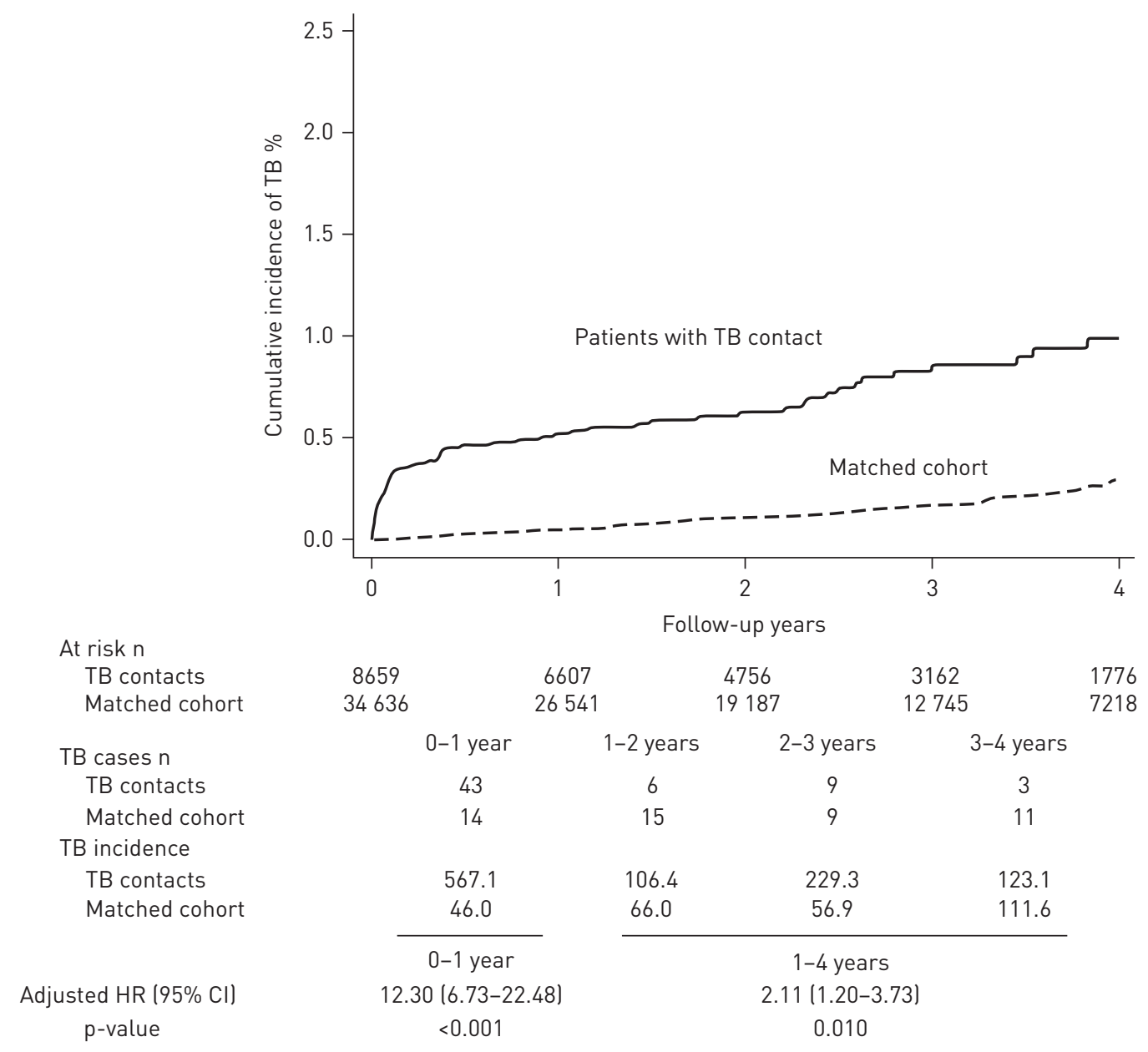

FIGURE 1 Kaplan-Meier analysis and annual incidence of tuberculosis (TB) occurrence in adult TB contacts and the matched cohort. The TB incidence is presented as cases per 100000 person-years. The hazard ratios in each follow-up period were adjusted for age, sex and comorbidities. The cumulative incidence of TB was significantly higher in TB contacts ( $\mathrm{p}<0.001$ by $\log$ rank test).

in figure 1. The annual TB incidence among TB contacts after exposure in the first, second, and third years were 567.1 cases, 106.4 cases, and 229.3 cases per 100000 person-years, respectively. The first year after the exposure showed the highest risk for active TB (adjusted HR 12.30, 95\% CI 6.73-22.48), and the adjusted HRs from year two to year four were 2.11 (95\% CI 1.20-3.73). In multivariate analysis, the independent risk factors associated with active TB development among adult TB contacts were age $\geqslant 65$ years old (HR 2.15, 95\% CI 1.22-3.81), male sex (HR 1.74, 95\% CI 1.07-2.81), and having autoimmune diseases (HR 2.60, 95\% CI 1.40-4.81), chronic obstructive pulmonary disease (COPD) (HR 1.95, 95\% CI 1.16-3.27) or liver cirrhosis (HR 2.61, 95\% CI 1.03-6.65).

Analysing the trend of active TB development after exposure helps to improve our contact investigation strategy. In a large meta-analysis that included more than 200 studies, Fox et al. [2] reported that the incidence rate ratio (IRR) among contacts during the first year was 46.6 (IQR 3.2-68.0) in high-income countries and 15.9 (IQR 2.6-21.4) in low- to middle-income countries. In their meta-analysis, the IRR dropped in the second year and rose again in the third year. Interestingly, a similar dynamic pattern was found in the present study. As the information used in this study was obtained from a health insurance database, many important factors associated with TB exposure were not available, such as the closeness of contact and smear positivity and disease severity of the index case. The limited number of cases with active TB after the first year also made it difficult to compare the TB incidence in each single year. However, when we combined the cases from year two to year four we still found that the incidence of active TB was significantly higher in adult TB contacts, with a HR of 2.11. Due to the limitations mentioned earlier, the dynamic pattern of active TB occurrence in the present study could come from reporting bias or chance. Nonetheless, we also speculate that it could be related to our current contact investigation strategy. This is based on current WHO recommendations [4] with active case-finding in the first year after exposure 
leading to early diagnosis of some TB cases, which in turn masks the incidence curve of active TB in the second year. As active case-finding stopped after the first year, the true impact of TB exposure on the TB incidence is shown in the third year. Our findings suggest the potential limitation of a 6-12-month follow-up for adult TB contacts. A well-designed, prospective study is required for verification.

Plenty of studies have identified several independent risk factors for LTBI and active TB among TB contacts [5, 8-12]. However, studies which focus on adult TB contacts, and especially on analysing underlying comorbidities that increase their susceptibility to active TB, are much more limited. To the best of our knowledge, the present study is the first to focus on adult TB contacts and include details of comorbidities for analysis. We found that older age, male sex, and having autoimmune diseases, COPD or liver cirrhosis were independent predictors for TB development among adult TB contacts. The depressed immunity related to autoimmune diseases and liver cirrhosis obviously increases the risk for active TB $[13,14]$. Animal and cellular studies have demonstrated the negative impact of smoke exposure on the local immunity of the lungs [15]. These risk factors are of pivotal importance and should never be overlooked in our practice of contact investigation.

TB contacts are high-risk individuals for active TB development and contact investigation is an effective way of active case-finding. This population-based cohort study demonstrated that the annual incidence of active $\mathrm{TB}$ in adult $\mathrm{TB}$ contacts is highest during the first year following exposure, but it also remains significantly higher after the first year as compared with a matched cohort. An intensive and frequent follow-up strategy in contact investigation is definitely needed during the first year after exposure. However, the increased risk of active TB after the first year should not be ignored and a risk-stratified strategy to extend the follow-up period in targeted adult TB contacts is needed. The underlying comorbidities that increase the risk of active TB development could be included in contact investigation and evaluation. contacts is needed http://ow.ly/I9t28

Jia-Yih Feng ${ }^{1,2,9}$, San-Chi Chen ${ }^{3,9}$, Ming-Che Lee ${ }^{1}$, Wen-Chien Fan ${ }^{4,5}$, Wei-Juin Su ${ }^{1,5}$, Yu-Wen Hu ${ }^{5,6}$, Chiu-Mei Yeh ${ }^{7}$, Tzeng-Ji Chen ${ }^{5,7}$ and Chia-Jen Liu ${ }^{3,8}$

${ }^{1}$ Dept of Chest Medicine, Taipei Veterans General Hospital, Taipei, Taiwan, R.O.C. ${ }^{2}$ Institute of Clinical Medicine, School of Medicine, National Yang-Ming University, Taipei, Taiwan, R.O.C. ${ }^{3}$ Division of Hematology and Oncology, Dept of Medicine, Taipei Veterans General Hospital, Taipei, Taiwan, R.O.C. ${ }^{4}$ Division of Infectious Disease, Dept of Medicine, Taipei Veterans General Hospital, Taipei, Taiwan, R.O.C. ${ }^{5}$ School of Medicine, National Yang-Ming University, Taipei, Taiwan, R.O.C. ${ }^{6}$ Cancer Center, Taipei Veterans General Hospital, Taipei, Taiwan, R.O.C. ${ }^{7}$ Dept of Family Medicine, Taipei Veterans General Hospital, Taipei, Taiwan, R.O.C. ${ }^{8}$ School of Medicine, Institute of Public Health, National Yang-Ming University, Taipei, Taiwan, R.O.C. ${ }^{9}$ Both authors contributed equally.

Correspondence: Chia-Jen Liu, Division of Hematology and Oncology, Dept of Medicine, Taipei Veterans General Hospital, No. 201 Shipai Rd., Sec. 2, Taipei, Taiwan 11217. E-mail: chiajenliu@gmail.com

Received: July 092014 | Accepted after revision: Jan 032015 | First published online: Feb 192015

Conflict of interest: None declared.

\section{References}

1 Diel R, Loddenkemper R, Zellweger JP, et al. Old ideas to innovate tuberculosis control: preventive treatment to achieve elimination. Eur Respir J 2013; 42: 785-801.

2 Fox GJ, Barry SE, Britton WJ, et al. Contact investigation for tuberculosis: a systematic review and meta-analysis. Eur Respir J 2013; 41: 140-156.

3 Erkens CG, Kamphorst M, Abubakar I, et al. Tuberculosis contact investigation in low prevalence countries: a European consensus. Eur Respir J 2010; 36: 925-949.

4 World Health Organization. Recommendations for investigating contacts of persons with infectious tuberculosis in low- and middle-income countries. Geneva, WHO Press, 2012. http://apps.who.int/iris/bitstream/10665/77741/1/ 9789241504492_eng.pdf

5 Aissa K, Madhi F, Ronsin N, et al. Evaluation of a model for efficient screening of tuberculosis contact subjects. Am J Respir Crit Care Med 2008; 177: 1041-1047.

6 Kuo SC, Chen YT, Li SY, et al. Incidence and outcome of newly-diagnosed tuberculosis in schizophrenics: a 12-year, nationwide, retrospective longitudinal study. BMC Infect Dis 2013; 13: 351.

7 Ou SM, Liu CJ, Chang YS, et al. Tuberculosis in myasthenia gravis. Int J Tuberc Lung Dis 2013; 17: 79-84.

8 Morán-Mendoza O, Marion SA, Elwood K, et al. Risk factors for developing tuberculosis: a 12-year follow-up of contacts of tuberculosis cases. Int J Tuberc Lung Dis 2010; 14: 1112-1119.

9 Chan PC, Shinn-Forng Peng S, Chiou MY, et al. Risk for tuberculosis in child contacts. Development and validation of a predictive score. Am J Respir Crit Care Med 2014; 189: 203-213.

10 Lienhardt C, Fielding K, Sillah J, et al. Risk factors for tuberculosis infection in sub-Saharan Africa: a contact study in The Gambia. Am J Respir Crit Care Med 2003; 168: 448-455.

11 Mendes MA, Gaio R, Reis R, et al. Contact screening in tuberculosis: can we identify those with higher risk? Eur Respir J 2013; 41: 758-760.

12 Narasimhan P, Wood J, Macintyre CR, et al. Risk factors for tuberculosis. Pulm Med 2013; 2013: 828939. 
14 Chang YS, Liu CJ, Ou SM, et al. Tuberculosis infection in primary Sjögren's syndrome: a nationwide population-based study. Clin Rheumatol 2014; 33: 377-383.

15 Shang S, Ordway D, Henao-Tamayo M, et al. Cigarette smoke increases susceptibility to tuberculosis - evidence from in vivo and in vitro models. J Infect Dis 2011; 203: 1240-1248.

\section{Suboptimal specificity of Xpert MTB/RIF among treatment-experienced patients}

\section{To the Editor:}

The Xpert MTB/RIF assay (Cepheid, Sunnyvale, CA, USA) is strongly recommended by the World Health Organization as an initial diagnostic test for treatment-experienced patients of any retreatment category [1-3]. Yet, retreatment tuberculosis (TB) suspects have been infrequently included in studies of Xpert [4], probably because current-generation PCR-based tests are unable to determine Mycobacterium tuberculosis viability [5]. Indeed, Xpert is known to frequently remain positive at the end of standard short-course therapy [6], with case reports emerging of Xpert false-positivity for up to 5 years post-treatment completion [7, 8]. Furthermore, 56\% $(\mathrm{n}=3485 / 6285)$ of specificity data informing the most recent Cochrane meta-analysis [4] was derived from validation and demonstration studies [9, 10], which may be optimistic due to selection bias related to post-enrolment exclusions [7]. Not surprisingly, there have been increasing calls to clarify the guidelines for use of Xpert among treatment-experienced patients [11].

To address these concerns, we prospectively enrolled individuals with a history of prior treatment in a high HIV prevalence, limited resource setting (Harare, Zimbabwe) over a 2 year (2011-2013) period. We hypothesised that among individuals with a history of prior treatment, specificity would be lower than that reported in pooled summaries and would correlate with mean cycle threshold (i.e. mycobacterial load) and time since prior treatment completion. All participants provided written informed consent, and ethical approval was obtained from the Medical Research Council of Zimbabwe and the University of California, San Francisco Human Research Protection Program. Notified cases were categorised according to the outcome of their most recent course of treatment [1] as either 1) "recurrent TB" (TB following cure or completion of treatment of a previous TB episode), or 2) "prevalent retreatment TB" (treatment failure, which was defined as acid-fast bacilli sputum smear-positivity at month 5 or later). The reference standard for M. tuberculosis detection was a positive result on solid (Löwenstein-Jensen media), liquid (BBL MGIT Mycobacterial Growth Indicator Tubes (Becton Dickinson, Sparks, MD, USA)), or microscopic-observation drug-susceptibility (MODS) culture (TB MODS Kit; Hardy Diagnostics, Santa Maria, CA, USA) [12, 13]. Xpert false detection of active TB ("Xpert false-positive") was defined as Xpert-positivity in absence of any of the three culture modalities being positive. The Biomedical Research and Training Institute Tuberculosis Laboratory within the National Microbiology Reference Laboratory is a centre for Trials of Excellence in Southern Africa. The most recent Centre for American Pathologists assessment in 2014 demonstrated 100\% agreement for isoniazid, rifampicin, ethambutol and streptomycin resistance testing.

During the study, we enrolled 380 ambulatory retreatment TB cases, representing $\sim 65 \%$ of all retreatment TB cases notified to the Harare City Health Department during this time period. After excluding 37 patients who were not initiated on treatment, who were on second-line agents for known multidrug-resistant TB, or who did not have culture results available, 149 (43.4\%) patients had recurrent TB, and 194 (56.6\%) were prevalent retreatment cases. The diagnostic accuracy of Xpert for M. tuberculosis detection was evaluated among 149 patients with recurrent TB. Most (111 (74.5\%) out of 149) had HIV comorbidity with a median (interquartile range (IQR)) CD4 count of $177(83-350)$ cells $\cdot \mathrm{mm}^{-3}$. The median (IQR) time from completion of previous TB treatment to clinical re-presentation and Xpert testing was 19.6 (7.962.9) months. 127 (85.2\%) Xpert tests were generation 4. Of the 149 patients included in the analysis, 24 (16.1\%) had culture-positive rifampicin (RMP)-resistant TB; 65 (43.6\%) had culture-positive 